\title{
Efectos de los abonos orgánicos en el crecimiento de plantas de geranio y belén
}

\author{
Organic fertilizers effects on geranium and impatiens seedlings growth
}

\author{
Elena Huerta-Muñoz ${ }^{1}$ Javier Cruz-Hernández ${ }^{1 *}$
}

\begin{abstract}
RESUMEN
El procesamiento de estiércoles producidos en traspatios de comunidades rurales por compostaje o lombricompostaje representa una alternativa viable para elaborar abonos orgánicos; no obstante, es necesario comprobar que son adecuados como sustratos o componentes de sustratos para el buen desarrollo de especies cultivadas. Se evaluó el efecto de cuatro abonos orgánicos, uno comercial, un lombricompost y dos compost derivados de estiércol vacuno y ovino, aplicados al sustrato a proporciones de 75,50 y $25 \%$, en comparación con tierra de hoja, en el crecimiento y floración de plantas de geranio y belén, dos especies de importancia en los traspatios rurales. Se midió número de hojas, diámetro y longitud del tallo, crecimiento radicular, floración y materia seca en crecimiento, floración, y cosecha de ambas especies. Los datos obtenidos se analizaron mediante un modelo lineal general (GLM) y prueba de comparación de medias de Tukey $(p<0.05)$. Se encontró que, en general, los tratamientos con lombricompost de estiércol vacuno, resultaron muy favorables para el crecimiento, número de hojas en geranio, desarrollo radicular y floración de ambas especies. Por el contrario, el abono comercial y el compost de estiércol vacuno a proporciones superiores a $50 \%$ generaron efectos negativos en crecimiento vegetativo, radicular y floración, resultando más acentuados en belén.
\end{abstract}

PALABRAS CLAVE

compost, lombricompost, Pelargonium grandiflorum, Impatiens walleriana

\section{ABSTRACT}

The handling and processing of cattle manure produced in rural backyards by composting and vermicomposting techniques represents a suitable alternative to elaborate organic fertilizers. However, before these products are used or recommended is necessary to run some tests to assure their safe and adequate use as a growing medium or as a growing medium component for plants full development. The effects of two manure composts (bovine compost, ovine compost), a bovine lombricompost, and an organic commercial brand, were evaluated as substrates for the growth and production of geraniums and impatiens seedlings, both species have an important presence as ornamental plants in backyards. All fertilizers were applied at 75, 50 and $25 \%$ proportions; forest topsoil was used as control. The number of functional leaves, stem length, stem diameter, radicular growth, flowering and dry biomass were the variables evaluated using a general linear model (GLM) and the Tukey test $(p<0.05)$ when statistical differences were found. The results show that, in general, lombricompost treatments are very suitable to vegetative growth, number of leaves in geranium, radicular growth, flowering in both species development, while the commercial and the bovine compost produced a negative effect in both growth and flowering when $50 \%$ or higher doses were applied, with the most negative effect in impatiens seedlings.

KEYWORDS

compost, lombricompost, Pelargonium grandiflorum, Impatiens walleriana

\footnotetext{
${ }^{1}$ Programa en Estrategias para el Desarrollo Agrícola Regional. Colegio de Postgraduados, Campus Puebla. México.

* Autor para correspondencia. Boulevard Forjadores de Puebla 205, Santiago Momoxpan. 72760 San Pedro Cholula, Puebla, México.

Correo electrónico: javiercruz@colpos.mx
} 


\section{INTRODUCCIÓN}

En los traspatios de las comunidades rurales en México, y particularmente en diversos municipios del estado de Puebla, se liberan y acumulan diferentes estiércoles en los terrenos cercanos a las casas o unidades habitacionales (Guarneros-Zarandona et al. 2014). Estos estiércoles ocasionan malos olores, contaminación de aguas, suelo y pueden ser fuente de infecciones o enfermedades, cuyos efectos dependen de la cantidad, tipo de excreta, de la alimentación del ganado, etc. (FAO 2006, Pinos-Rodríguez et al. 2012).

La gestión o aprovechamiento de los estiércoles como medio eficiente de reciclaje en comunidades rurales mediante la elaboración de abonos orgánicos, como compost, lombricompost y lixiviados, ha representado una alternativa viable en diferentes experiencias para reducir la contaminación y obtener un insumo que le da valor agregado a los residuos en beneficio de los productores (Ansorena-Miner 2010, Preston 2005, Ramos y Terry 2014, Velásquez y León 2006).

Sin embargo, para que un abono orgánico sea recomendado para la producción de cultivos, debe reunir dos características. Por un lado, debe aportar nutrientes y materia orgánica; ser estable y maduro con bajo contenido de materiales indeseables o sustancias tóxicas (Íñiguez y Crohn 2010). Por otro lado, hay que garantizar que sea adecuado para los productores en sus sistemas de producción y en sus traspatios, por lo cual, al ser valorado o aplicado, debe generar efectos benéficos en el crecimiento y producción de los cultivos de importancia en las comunidades (Ansorena-Miner 2010, Preston 2005).

Por otra parte, en los traspatios se conservan y cultivan diferentes especies de anuales, hortalizas, frutales, medicinales $\mathrm{u}$ ornamentales (Vieyra et al. 2004). La diversidad de especies en los traspatios es diferente en cada zona o región. Al respecto, en un estudio realizado por Guarneros-Zarandona et al. (2014), se observó en traspatios una diversidad específica de 130 especies; donde la mayor proporción la representan las ornamentales (57 especies) de las familias Rosaceae, Araceae, Lilaceae y Asteraceae (44\% del total). Las plantas ornamentales presentan importancia social, cultural y económica, porque se intercambian entre las familias, se utilizan en rituales $\mathrm{y}$ fiestas religiosas o se comercializan en mercados locales y regionales.

Con el objetivo de determinar el efecto de abonos orgánicos derivados de residuos locales sobre el crecimiento de cultivos ornamentales, en la presente in- vestigación se evaluó el efecto de un abono comercial, un lombricompost y dos composts derivados de estiércoles de vacuno y ovino aplicados a proporciones de 75,50 y $25 \%$ en el sustrato y en comparación con tierra de hoja, sobre el crecimiento y floración de plantas de belén y geranio, especies de importancia en traspatios de comunidades rurales.

\section{Materiales Y MÉTODOS}

\section{Sitio de estudio}

Los experimentos se realizaron en las instalaciones del Colegio de Postgraduados, Unidad Huejotzingo, en el área de invernaderos y de malla-sombra, durante los meses de junio a octubre de 2013.

\section{Características fisicoquímicas de los abonos orgánicos}

En el cuadro 1 se presentan las características de los abonos sujetos a evaluación agronómica en el presente estudio.

\section{Material vegetal}

Las especies de ornamentales seleccionadas para los experimentos fueron: geranio (Pelargonium grandiflorum Willd [Geraniaceae]), variedad 485, Diablo Rojo; y belén (Impatiens walleriana Hook.f. [Balsaminaceae]), variedad 334, Super Sonic Red. Se trasplantaron esquejes enraizados de un promedio de $5 \mathrm{~cm}$ de altura y 6 hojas para geranios, y esquejes de $4 \mathrm{~cm}$ de altura y 10 hojas en promedio para belenes.

\section{Diseño de tratamientos y diseño experimental}

Los factores y niveles de estudio evaluados en el experimento, en cada especie indicada, se muestran en el cuadro 2.

A partir de la combinación de factores y niveles de estudio, en el cuadro 3 se indica la composición de los tratamientos evaluados.

La unidad experimental consistió en una maceta de 6 pulgadas con $1.5 \mathrm{~L}$ de sustrato y una planta o esqueje. Así, se tuvo un total de 140 unidades experimentales: 70 por cada especie (figura 1).

Se utilizó un diseño completamente al azar y se realizaron cinco repeticiones para cada uno de los 14 tratamientos evaluados, tanto en geranios como en belenes. El estudio tuvo una duración de 17 semanas. 
Cuadro 1. Propiedades físicas y químicas de los abonos analizados.

\begin{tabular}{|c|c|c|c|c|c|c|c|}
\hline \multirow[b]{2}{*}{ Tratamiento } & \multicolumn{2}{|c|}{ Propiedades físicas } & \multicolumn{5}{|c|}{ Propiedades químicas } \\
\hline & Gra (mm) & $\mathrm{DA}\left(\mathrm{g} \mathrm{cm}^{-3}\right)$ & $\mathrm{CE}\left(\mathrm{dS} \mathrm{m}^{-1}\right)$ & $\mathrm{pH}$ & Cen $(\%)$ & MO (\%) & $\mathrm{CO}(\%)$ \\
\hline Compost comercial & $0.5-4$ & 0.6 & 3.5 & 9.9 & 56.5 & 56.5 & 32.8 \\
\hline Compost vacuno & $0.5-4$ & 0.8 & 0.6 & 8.5 & 49.6 & 49.6 & 28.8 \\
\hline Compost ovino & $0.5-4$ & 0.6 & 1.4 & 9.1 & 35.2 & 35.2 & 20.5 \\
\hline $\begin{array}{l}\text { Lombricompost } \\
\text { vacuno }\end{array}$ & $0.5-4$ & 0.5 & 1.2 & 7.5 & 33.0 & 35.0 & 30.0 \\
\hline
\end{tabular}

Gra: granulometría; DA: densidad aparente; CE: conductividad eléctrica; Cen: cenizas; MO: materia orgánica; CO: carbono orgánico.

Cuadro 2. Factores y niveles de estudio de los tratamientos.

\begin{tabular}{ll}
\multicolumn{1}{c}{ FActor } & \multicolumn{1}{c}{ Nivel DE ESTUdio } \\
\hline Abono & Compost vacuno, \\
& lombricompost vacuno, \\
& compost ovino, comercial \\
Proporción $(\mathrm{v}: \mathrm{v})$ & 25,50 y $75 \%$ en mezcla con \\
& turba \\
\hline
\end{tabular}

Cuadro 3. Descripción de los tratamientos.

\begin{tabular}{|c|c|c|}
\hline Tratamiento & Авоno & $\begin{array}{c}\text { Proporción } \\
\text { (v:v) }\end{array}$ \\
\hline 1 (testigo) & tierra de hoja-suelo & $75: 25$ \\
\hline 2 & turba-agrolita & $75: 25$ \\
\hline 3 & comercial-turba & $75: 25$ \\
\hline 4 & comercial-turba & $50: 50$ \\
\hline 5 & comercial-turba & $25: 75$ \\
\hline 6 & $\begin{array}{l}\text { compost vacuno- } \\
\text { turba }\end{array}$ & $75: 25$ \\
\hline 7 & $\begin{array}{l}\text { compost vacuno- } \\
\text { turba }\end{array}$ & $50: 50$ \\
\hline 8 & $\begin{array}{l}\text { compost vacuno- } \\
\text { turba }\end{array}$ & $25: 75$ \\
\hline 9 & $\begin{array}{l}\text { lombricompost } \\
\text { vacuno-turba }\end{array}$ & $75: 25$ \\
\hline 10 & $\begin{array}{l}\text { lombricompost } \\
\text { vacuno-turba }\end{array}$ & $50: 50$ \\
\hline 11 & $\begin{array}{l}\text { lombricompost } \\
\text { vacuno-turba }\end{array}$ & $25: 75$ \\
\hline 12 & $\begin{array}{l}\text { compost ovino- } \\
\text { turba }\end{array}$ & $75: 25$ \\
\hline 13 & $\begin{array}{l}\text { compost ovino- } \\
\text { turba }\end{array}$ & $50: 50$ \\
\hline 14 & $\begin{array}{l}\text { compost ovino- } \\
\text { turba }\end{array}$ & $25: 75$ \\
\hline
\end{tabular}

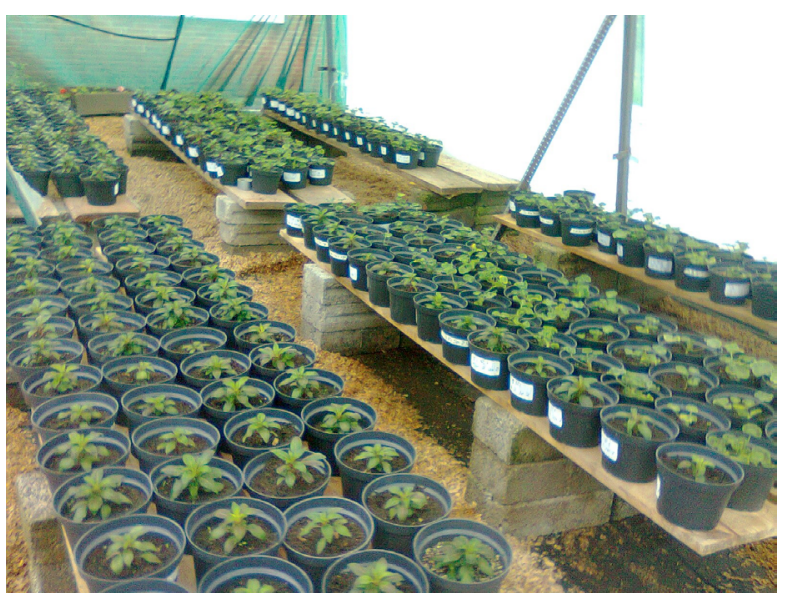

Figura 1. Unidades experimentales.

Por otro lado, como parte del manejo de cultivo, las plantas de ambas especies se regaron cada tercer día hasta el punto de saturación del sustrato. No se realizó fertilización química en ninguna de las etapas de desarrollo de las plantas. Veinte días después del trasplante, se realizó un despunte del tallo principal de cada planta en ambas especies para estimular formación de brotes vegetativos y florales laterales. Para prevenir la presencia de plagas y enfermedades, se aplicaron regularmente productos o extractos orgánicos comerciales a base de ajo y neem en concentraciones de $2.5 \mathrm{ml} / \mathrm{l}$ de agua. Para evitar competencia, el cultivo se mantuvo libre de malezas con eliminación manual de manera regular durante todo el ciclo de cultivo.

\section{Variables respuesta de crecimiento, floración y materia seca de plantas}

Las variables evaluadas fueron altura de planta y diámetro de tallos principales, longitud total de 
raíces, número de hojas funcionales, número de inflorescencias, número de botones florales, materia fresca y seca de raíz, tallo, hojas, inflorescencias y botones florales. La altura de planta se midió en centímetros con un flexómetro. El diámetro de tallos principales se registró en milímetros, medido con vernier (Digital Truper Stainless Stell ${ }^{\circledR}$ ) a $1.5 \mathrm{~cm}$ de la base de cada planta. El número de hojas funcionales se realizó por conteo en cada especie. Estas mediciones se realizaron cada quince días a partir de la segunda semana después del trasplante y hasta el día de la cosecha de las plantas.

Al final del experimento, se midió la longitud total de raíces de cada planta en centímetros con un flexómetro. Las variables de floración se determinaron por conteo de botones florales y flores en belenes, así como por número de inflorescencias en geranios. La materia fresca de raíz, tallo, hojas, inflorescencias y botones florales se determinó en una balanza (PG5001 METTLER TLEDO ${ }^{\circledR}$ 0.01) y se registró en gramos. Las muestras frescas fueron colocadas en una estufa de aire forzado (PRECISION Mechanical Convection Oven ${ }^{\circledR}$ ) a $70^{\circ} \mathrm{C}$ durante 72 horas con el fin de extraerles toda el agua y medir materia seca de las muestras. El cálculo de la materia seca se realizó con la fórmula siguiente:

$$
M S=\frac{P^{\prime}}{P} X 100
$$

donde:

$M S$ : materia seca

$P^{\prime}$ : peso de la muestra después de la desecación

$P$ : peso de la muestra antes de la desecación

100: para referir los resultados en porcentaje

Los resultados de las variables indicadas se presentan por separado en cada una de las especies estudiadas.

\section{Análisis estadístico de los datos}

Los datos obtenidos de las variables evaluadas se analizaron mediante un modelo lineal general (GLM) y prueba de comparación de medias Tukey $(p<0.05)$ cuando hubo diferencias estadísticamente significativas. El análisis estadístico se realizó con el programa $\mathrm{SAS}^{\circledR}$, versión 9. Los datos en porcentaje o en proporción se transformaron según las fórmulas indicadas por Montgomery (2011), antes de la ejecución del análisis estadístico.

\section{RESUlTADOS Y DISCUSIÓN}

\section{Variables de crecimiento en geranio}

La figura 2 muestra el efecto de los sustratos sobre la altura de las plantas. Los resultados evidencian que el testigo (tierra de hoja-suelo 75:25) tuvo efectos benéficos sobre los geranios y propició que alcanzaran buena altura; esto fue similar a lo ocurrido con la mayoría de los tratamientos, excepto T3 (comercial) y T6 (compost vacuno) aplicados a proporciones 75:25, donde se registraron los valores más bajos. En el caso de los abonos comercial y vacuno, es notorio que, entre más pequeña la proporción del compost, mayores son los valores de altura de la planta.

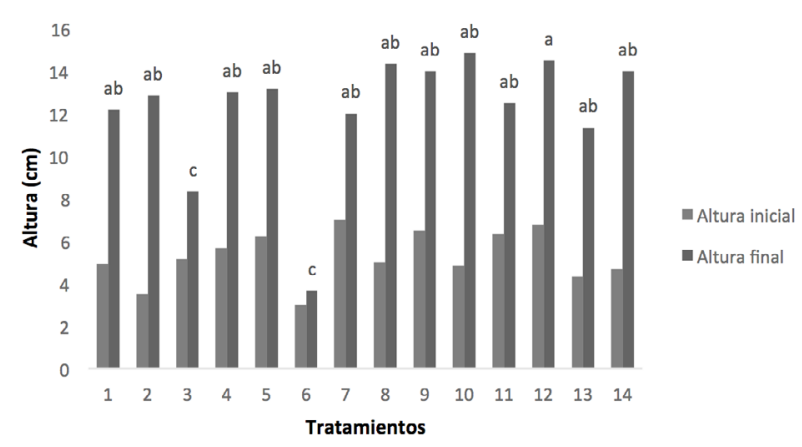

Figura 2. Efecto de abonos orgánicos en la altura de plantas de geranio. letras sobre las barras corresponden a la prueba de medias de Tukey $(p \leq 0.05)$.

Para la variable número de hojas funcionales, la diferencia más notoria se observó en los tratamientos T3 (comercial-turba 75:25) y T6 (compost de estiércol vacuno-turba 75:25). En ambos casos el número de hojas fue el más bajo en comparación con el resto de los tratamientos (figura 3). Por su parte, el testigo o T1 (tierra de hoja-suelo 75:25) provocó la emergencia sostenida de hojas con un promedio de 51; sin embargo, la mayor cantidad se obtuvo con T9 (lombricompost de vacuno-turba 75-25) y T11 (lombricompost de vacuno-turba 25:75) con un total de 61 y 62 hojas, respectivamente. Los tratamientos a base de lombricompost de estiércol vacuno obtuvieron los valores más sobresalientes.

Por otro lado, entre los tratamientos hechos a base de compost vacuno (T6 y T7) la producción de hojas fue relativamente pobre, excepto en T8 (compost vacuno: turba 25:75), en comparación con lo ocurrido cuando se utilizaron proporciones de 75:25 y 50:50. De manera similar a lo observado en la variable altura de planta, los tratamientos de compost comercial 


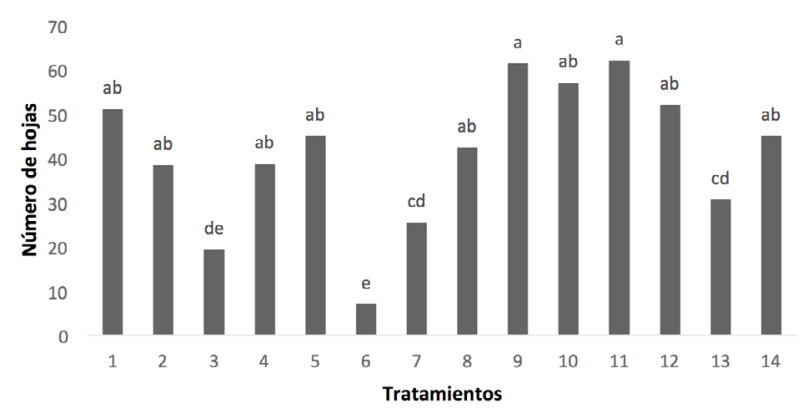

Figura 3. Efecto de abonos orgánicos en el número de hojas de geranio. Letras sobre las barras corresponden a la prueba de medias de Tukey $(p \leq 0.05)$.

y vacuno mostraron mejores resultados cuando se aplicó la proporción baja (25:75) en el sustrato.

En relación con el efecto en el crecimiento de la raíz, T11 (lombricompost de vacuno-turba 25:75) fue el tratamiento con el que se obtuvo la mayor elongación radicular, con longitud final de $65 \mathrm{~cm}$; los dos tratamientos restantes de lombricompost también permitieron buen desarrollo de este órgano (figura 4). El tratamiento 2 también mostró excelente desarrollo radicular; este tratamiento sobresale entre los abonos procesados, lo cual se atribuye al tamaño de los poros que se presentan en el sustrato cuando se utiliza agrolita. Nuevamente, el tratamiento 6 fue el que registró efectos más nocivos pues restringió del todo el desarrollo de la raíz.

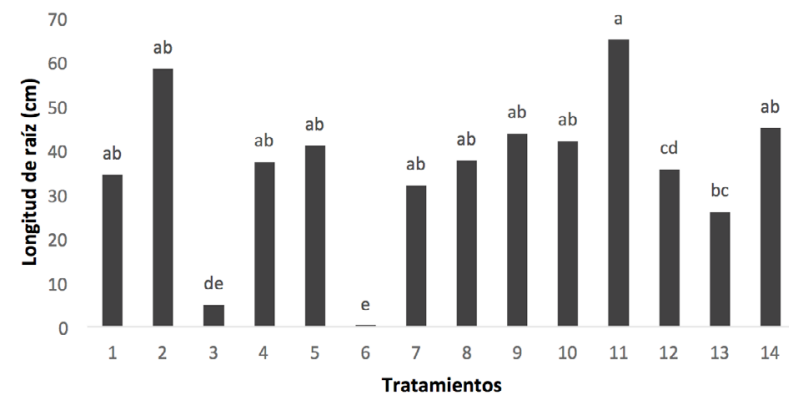

Figura 4. Efecto de abonos orgánicos en longitud de raíz de geranio. Letras sobre las barras corresponden a la prueba de medias de Tukey $(p \leq 0.05)$.

Por otra parte, en la variable diámetro de tallo, el tratamiento 12 registró el mayor engrosamiento en el cultivo de geranio (figura 5). Como se puede observar, los tallos adquirieron un mayor grosor al final del experimento. Si bien el engrosamiento fue lento o llegó incluso a detenerse de una toma de datos a otra, las plantas respondieron positivamente a casi todos los tratamientos, con excepción de dos: T6 que se mantuvo en $0.5 \mathrm{~mm}$ y T3.

Es de resaltar que los efectos negativos de los tratamienos 3 y 6 se reflejan en todas las variables de crecimiento evaluadas. En este sentido, el compost comercial tiene la mayor cantidad de materia orgánica, pero con valores de $\mathrm{pH}$ y conductividad eléctrica muy altos. Los efectos negativos asociados a su aplicación en altas proporciones sugieren que el material podría no estar maduro, o bien asociarse a las mismas fuentes de origen de este abono.

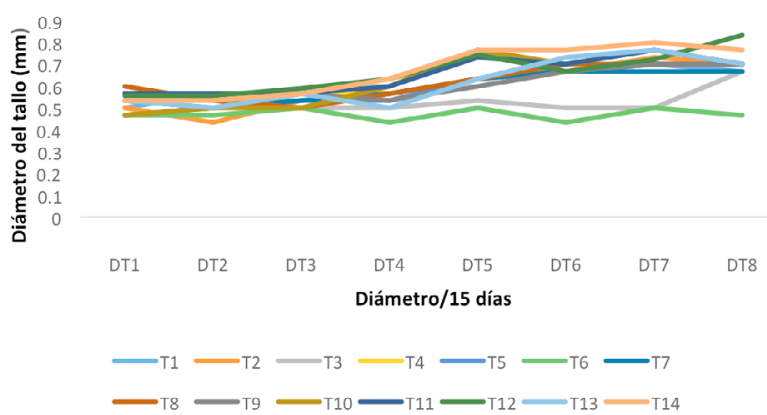

Figura 5. Efecto de abonos orgánicos en diámetro del tallo de geranio.

En contraste, en el caso del compost de vacuno, si bien el $\mathrm{pH}$ es alcalino, su valor es menor que el del abono comercial, pero sus efectos resultaron más nocivos al aplicarse en la misma dosis que este último, particularmente en el caso de las raíces, lo que podría explicarse por su elevada DA. La densidad aparente del compost de vacuno es la más alta entre todos los abonos sujetos a evaluación (0.8). Debido a ello, el desarrollo de raíces resultó afectado y, como consecuencia, el de toda la planta cuando se utilizó la porporción 75:25. Posiblemente, la baja porosidad ocasiona la reducción del potencal de crecimiento radicular y afecta la disponibilidad de oxígeno, así como la capacidad de retención y disponibilidad del agua.

Tanto en el compost comercial como el de estiércol vacuno, las proporciones aplicadas al 50:50 y 25:75 permitieron el crecimiento de los geranios aunque con valores menores a los lombricompost o a los tratamientos elaborados con compost de ovino. Cabe resaltar que, en el caso de estos últimos, los valores más bajos que se obtuvieron fueron al aplicar la proporción 50:50 (T13), en comparación con la proporción 75:25 (T12) y 25:75 (T14), lo cual podría deberse a interacciones ocurridas en la maceta. De manera general, con estos abonos los resultados fueron satisfactorios. 
El buen desarrollo de las plantas de geranio con los tratamientos de lombricompost se debe a que este abono posee un $\mathrm{pH}$ en el intervalo del neutro (6.5-7.5) y una DA baja. Si bien la conductividad eléctrica (CE) es más elevada en comparación con la del compost de vacuno, se sabe que los primeros presentan un mayor contenido de nutrientes que los compost. Estos resultados concuerdan con lo expresado por Santamaria et al. (2001), quienes refieren que, aunque las características químicas (y microbiológicas) de los compost y lombricompost son muy semejantes, la respuesta de los cultivos a la aplicación de este último suele ser superior a la de la composta convencional. Shirin et al. (2014) encontraron que tanto el desarrollo de la planta como la floración en geranios son mejores cuando se aplica lombricompost. Los autores mencionan que en su experimento, el diámetro del tallo, por ejemplo, fue mejor en las variedades de geranio cuando las dosis de aplicación fueron de 50 y $75 \%$, mientras que, en el presente trabajo, la tendencia fue que el mejor desarrollo de las plantas se dio cuando se utilizó la proporción más baja. Por su parte, Joshi et al. (2015) evaluaron el efecto de este tipo de abono elaborado con estiércol sobre plantas de geranio y encontraron aumentos en la germinación de semillas, peso del tallo, número y peso de hojas funcionales, contenido de clorofila, etcétera. De acuerdo con su experimento, los autores proponen eliminar por completo el uso de fertilizantes químicos. El lombricompost evaluado registró efectos similares o superiores a aquellos obtenidos con tierra de hoja.

\section{Variables de floración y materia seca en geranio}

En la variable número de inflorescencias por planta, se aprecia que, con el tratamiento T5 comercial-turba 25:75 y con T9 lombricompost de vacuno-turba 75:25, se obtuvieron los valores más altos (figura 6). De manera contrastante, el tratamiento 3 sólo arrojó una inflorescencia en promedio durante la etapa de evaluación, mientas que, con el tratamiento 6, no se presentó floración.

Como se puede apreciar en el cuadro 4, los mayores valores de peso seco tanto en raíz como en tallo se observaron en T11 (lombricompost de vacuno-turba 25:75) con 8.86 y con $5.53 \mathrm{~g}$, respectivamente; el tratamiento 9 (lombricompost vacuno-turba 75:25) fue el que obtuvo mayor valor de materia seca en hojas con $6.70 \mathrm{y}$, en el caso de las inflorecencias, fue T5 (comercial: turba 25:75) con 2.43 el del máximo valor. De manera similar a lo observado en las variables anteriores, los valores más bajos se obtuvieron con T3 y T6.

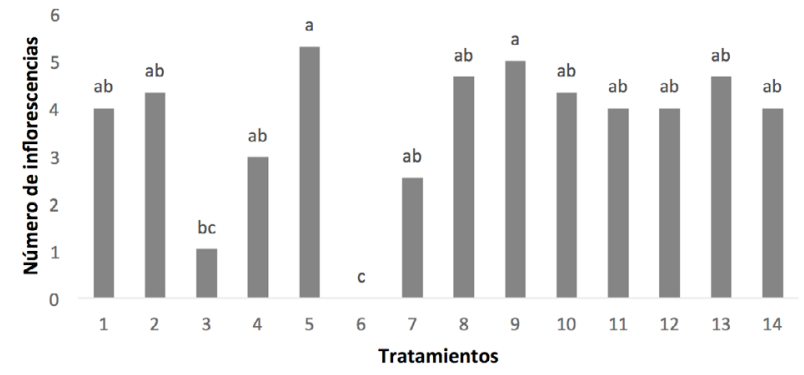

Figura 6. Efecto de abonos orgánicos en el número de inflorescencias de geranio. Letras sobre las barras corresponden a la prueba de medias de Tukey $(p \leq 0.05)$.

Cuadro 4. Comparación de medias por tratamiento en variables de materia seca en geranio.

\begin{tabular}{cllll}
\hline TrATAMIENTO & PSR (g) & PST (g) & PSH (g) & PSI (g) \\
\hline 1 & $2.26^{\mathrm{abc} *}$ & $3.86^{\mathrm{ab}}$ & $5.50^{\mathrm{ab}}$ & $1.60^{\mathrm{ab}}$ \\
2 & $3.10^{\mathrm{ab}}$ & $4.53^{\mathrm{a}}$ & $3.43^{\mathrm{bcd}}$ & $1.30^{\mathrm{ab}}$ \\
3 & $0.13^{\mathrm{c}}$ & $1.80^{\mathrm{ab}}$ & $1.43^{\mathrm{de}}$ & $0.26^{\mathrm{b}}$ \\
4 & $1.50^{\mathrm{abc}}$ & $2.76^{\mathrm{ab}}$ & $3.40^{\mathrm{bcd}}$ & $1.20^{\mathrm{ab}}$ \\
5 & $1.36^{\mathrm{bc}}$ & $3.60^{\mathrm{ab}}$ & $4.90^{\mathrm{abc}}$ & $2.43^{\mathrm{a}}$ \\
6 & $0.00^{\mathrm{c}}$ & $0.23^{\mathrm{b}}$ & $0.13^{\mathrm{e}}$ & $0.00^{\mathrm{b}}$ \\
7 & $1.30^{\mathrm{bc}}$ & $2.70^{\mathrm{ab}}$ & $2.06^{\mathrm{cde}}$ & $1.06^{\mathrm{ab}}$ \\
8 & $2.16^{\mathrm{abc}}$ & $4.70^{\mathrm{a}}$ & $3.90^{\mathrm{abcd}}$ & $1.60^{\mathrm{ab}}$ \\
9 & $1.56^{\mathrm{abc}}$ & $3.23^{\mathrm{ab}}$ & $6.70 \mathrm{a}$ & $1.83^{\mathrm{ab}}$ \\
10 & $1.83^{\mathrm{abc}}$ & $3.60^{\mathrm{ab}}$ & $4.73^{\mathrm{abc}}$ & $1.73^{\mathrm{ab}}$ \\
11 & $8.86^{\mathrm{a}}$ & $5.53^{\mathrm{a}}$ & $4.03^{\mathrm{abcd}}$ & $1.03^{\mathrm{ab}}$ \\
12 & $1.40^{\mathrm{abc}}$ & $3.10^{\mathrm{ab}}$ & $3.90^{\mathrm{abcd}}$ & $1.65^{\mathrm{ab}}$ \\
13 & $0.56^{\mathrm{c}}$ & $1.53^{\mathrm{ab}}$ & $2.63^{\mathrm{bcde}}$ & $1.30^{\mathrm{ab}}$ \\
14 & $2.43^{\mathrm{abc}}$ & $5.46^{\mathrm{a}}$ & $5.23^{\mathrm{ab}}$ & $1.63^{\mathrm{ab}}$ \\
\hline
\end{tabular}

* Grupos de letras distintas en la misma columna indican diferencias significativas, según la prueba de Tukey $(p \leq 0.05)$. PSR: peso seco de raíz; PST: peso seco del tallo; PSH: peso seco de hojas; PSI: peso seco de inflorescencias.

Al respecto, Grigatti et al. (2007), al evaluar 25, 50,75 y $100 \%$ de compost, indican que la respuesta depende de la especie, pero la mayor floración, materia seca y crecimiento se presenta a proporciones de 25 a $50 \%$, con reducciones en el desarrollo y alteraciones en la nutrición cuando se aplican proporciones superiores a $50 \%$, con lo que pueden generarse efectos antagónicos entre nutrientes con aplicaciones elevadas de compost, resultados similares a los observados en el presente estudio con proporciones 
de $25 \%$. Por su parte, Chamani et al. (2008) mencionan que las mejores respuestas en floración en begonia se obtienen con lombricompost aplicado a $20 \%$ en el sustrato, mientras que García-Gomez et al. (2002) coinciden en indicar que la respuesta depende de la especie vegetal como del tipo de compost y la porporción aplicada, y establecen que los aportes de $\mathrm{N}$ y K con los composts, así como el porcentaje de poros, la conductividad eléctrica (CE) y la concentración de cloro en el sustrato son los principales factores que influyen en el crecimiento y desarrollo de caléndula y calceolaria.

\section{Variables de crecimiento en plantas de belén}

La figura 7 muestra una comparación del efecto de los diferentes abonos en la altura de las plantas. Los resultados evidencian que el testigo T1 (tierra de hoja-suelo 75:25) tuvo efectos benéficos sobre las plantas, similares a los de la mayoría de los tratamientos, excepto con los tratamientos T3 (comercial-turba 75:25) y T6 (compost de vacuno-turba 75:25), donde se generaron efectos negativos en esta variable. Asimismo, también el compost de vacuno-turba 50:50 (T7) afectó el desarrollo de las pantas de belén.

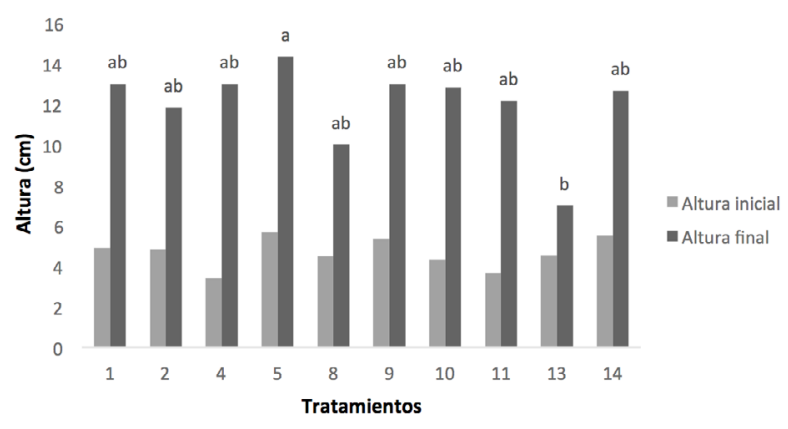

Figura 7. Efecto de abonos orgánicos en altura de plantas de belén. Letras sobre las barras corresponden a la prueba de medias de Tukey $(p \leq 0.05)$.

Estos resultados concuerdan con lo reportado por Atiyeh et al. (2001), quienes refieren que las cantidades moderadas de compost, entre 10 y $40 \%$, son las adecuadas para obtener beneficios en los cultivos, mientras que con concentraciones más altas, los efectos pueden llegar a ser nocivos. Resultados similares fueron presentados por Lazcano y Domínguez (2010), quienes observaron efectos negativos en el crecimiento de plantas con proporciones mayores a $40 \%$.

El follaje y crecimiento de la raíz de las plantas puede verse afectado por la proporción y tipo de abono usado en el sustrato. Sin embargo, en las variables de emisión de hojas funcionales y en longitud de raíz en plantas de belén, no se observaron diferencias significativas entre tratamientos (figuras 8 y 9), en contraste con lo observado en las plantas de geranio. Por lo anterior, la respuesta a los tipos y proporciones usadas de abonos dependen de la especie vegetal en estudio, como lo indican García-Gomez et al. (2002) y Grigatti et al. (2007).

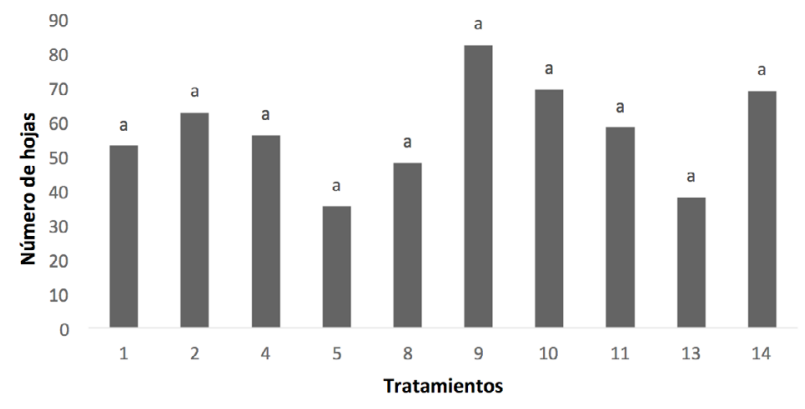

Figura 8. Efecto de abonos orgánicos en el número de hojas de plantas de belén. Letras sobre las barras corresponden a la prueba de medias de Tukey $(p \leq 0.05)$.

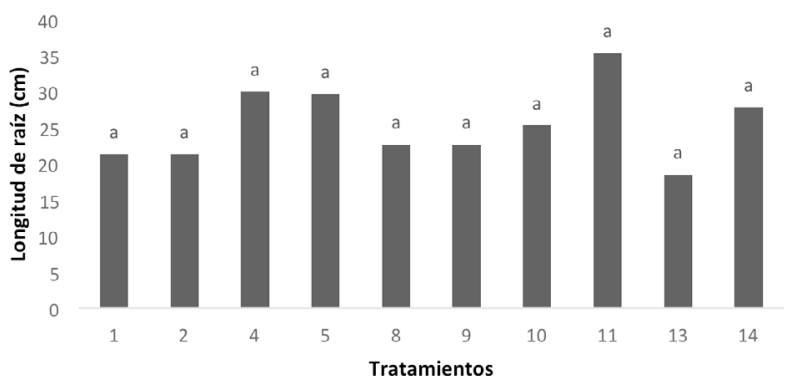

Figura 9. Efecto de abonos orgánicos en longitud de raíz de plantas de belén. Letras sobre las barras corresponden a la prueba de medias de Tukey $(p \leq 0.05)$.

Al analizar la variable diámetro de tallo de las plantas de belén, se observó que el tallo principal aumentó y posteriormente se mantuvo entre la toma 2 y la 4 en la mayoría de los tratamientos (figura 10). El tratamiento 11 propició mayor diámetro en el tallo principal y las plantas lograron buen desarrollo de la parte aérea.

Se distingue que, en belén, la proporción de los abonos en la mezcla del sustrato tuvo mayor impacto sobre el desarrollo de las plantas en comparación con el efecto observado en los geranios. En este sentido, los tratamientos 3, 6 y 12 ocasionaron la muerte de la planta, todos con proporción 75:25 en relación compost-turba. Asimismo, el tratamiento 7 (compost vacuno-turba) en relación 50:50 tuvo un efecto negativo 


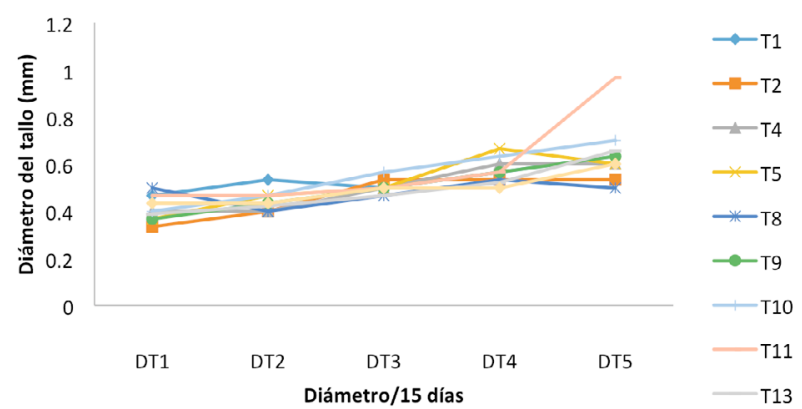

Figura 10. Efecto de abonos orgánicos en diámetro del tallo de plantas de belén.

en el crecimiento de la planta. Estos efectos pueden deberse a los valores de $\mathrm{pH}$ y $\mathrm{CE}$ en el caso del compost comercial (T3) y al pH y DA en el caso del compost vacuno (T6). Dado que la planta sólo se desarrolló con la dosis más baja del compost vacuno (T7), el belén puede ser más susceptible a los valores de densidad aparente. En comparación con la proporción 75:25, la proporción 50:50 y 25:75 de compost comercial (T4 y T5) y de 25:75 en compost de vacuno (T8) permitieron el desarrollo de las plantas, aunque en ambos casos el crecimiento resultó menor al promovido por el resto de los tratamientos.

En el caso de los tratamientos con lombricompost, las variables que mostraron mayor respuesta a este material fueron la longitud de raíz y el diámetro del tallo. Asciutto et al. (2006) evaluaron el efecto de diferentes lombricompost como promotores de crecimiento y de sanidad en plantas de belén y hallaron que los tratamientos en proporciones de 75 y 100\% mostraron incrementos importantes en el área foliar, altura de la planta y pesos fresco y seco de parte aérea y raíz. Por su parte, García-Albarado et al. (2010) evaluaron el efecto como sustratos de composts elaborados a partir de estiércol bovino, aplicados a diferentes proporciones junto con suelo agrícola en petunias (Petunia $x$ hybrida Hort. Vilm. [Andr]). Encontraron que el mejor sustrato para su producción era el que contenía 30\% de compost en comparación con aquel a $80 \%$, ya que con la dosis más alta se consiguieron efectos negativos sobre las plantas ornamentales.

\section{Variables de floración y materia seca en plantas de belén}

Los botones florales fueron producidos en mayor cantidad en el tratamiento T1 (figura 11). Por otro

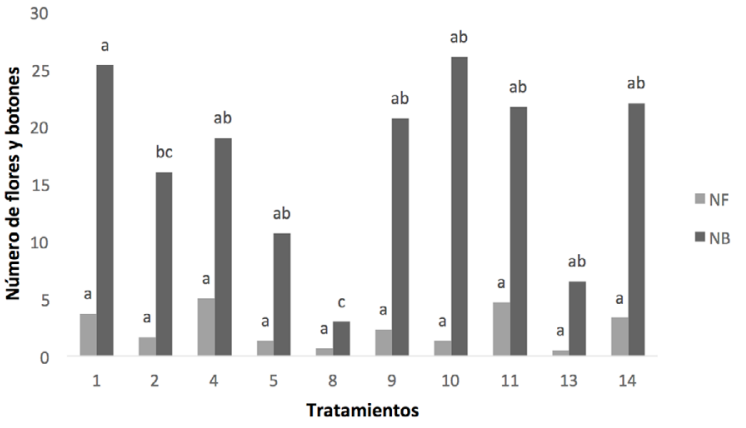

Figura 11. Efecto de abonos orgánicos en el número de flores y brotes de plantas de belén. Letras sobre las barras corresponden a la prueba de medias de Tukey $(p \leq 0.05)$.

lado, el valor más bajo se observó en el T8 (compost vacuno-turba 25:75). La evidencia muestra efectos benéficos con el lombricompost, similares a los observados con tierra de hoja, comúnmente usada en la propagación de plantas ornamentales.

Como se muestra en el cuadro 5, los mayores valores de peso seco de raíz se obtuvieron con T2 (turba-agrolita 75:25) con 21.2 g; y para botones florales con T1 (tierra de hoja-tierra 75:25) con $3.70 \mathrm{~g}$.

Cuadro 5. Comparación de medias por tratamiento en variables de materia seca en belén.

\begin{tabular}{ccc}
\hline Tratamiento & PSR $(\mathbf{g})$ & PSB $(\mathbf{g})$ \\
\hline 1 & $14.95^{\mathrm{abc} *}$ & $3.70^{\mathrm{a}}$ \\
2 & $21.25^{\mathrm{a}}$ & $1.30^{\mathrm{ab}}$ \\
3 & $14.00^{\mathrm{abcd}}$ & $1.10^{\mathrm{ab}}$ \\
4 & $8.50^{\mathrm{bcde}}$ & $0.30^{\mathrm{b}}$ \\
5 & $4.60^{\mathrm{de}}$ & $0.16^{\mathrm{b}}$ \\
6 & $17.30^{\mathrm{ab}}$ & $1.06^{\mathrm{ab}}$ \\
7 & $8.00^{\mathrm{bcde}}$ & $1.05^{\mathrm{ab}}$ \\
8 & $8.00^{\mathrm{bcde}}$ & $1.16^{\mathrm{ab}}$ \\
9 & $2.1^{0 \mathrm{e}}$ & $0.20^{\mathrm{b}}$ \\
10 & $5.00^{\mathrm{cde}}$ & $1.10^{\mathrm{ab}}$ \\
11 & $8.86^{\mathrm{a}}$ & $5.53^{\mathrm{a}}$ \\
12 & $1.40^{\mathrm{abc}}$ & $3.10^{\mathrm{ab}}$ \\
13 & $0.56^{\mathrm{c}}$ & $1.53^{\mathrm{ab}}$ \\
14 & $2.43^{\mathrm{abc}}$ & $5.46^{\mathrm{a}}$ \\
\hline
\end{tabular}

* Grupos de letras distintas en la misma columna indican diferencias significativas, según la prueba de Tukey $(p \leq 0.05)$. PSR: peso seco de raíz; PSB: peso seco botones florales. Los tratamientos $3,6,7$ y 12 no aparecen por ausencia de datos por efecto de proporción alta de abono usado en el sustrato.

Aplicaciones de 20\% de lombricompost de estiércol han generado los mejores efectos en 
desarrollo y floración de petunia (Chamani et al., 2008). En contraste con los resultados obtenidos en el presente estudio, Íñiguez y Crohn (2010) indican que porporciones de 70 a $100 \%$ de compost de desechos de rastro no mostraron efectos fitotóxicos ni daños en la raíz, desarrollo y floración de plantas de geranio, resultados que pueden estar asociados al grado de madurez de los abonos utilizados, a la especie vegetal y a las diferentes fuentes orgánicas utilizadas (Durán y Henríquez, 2007).

Con el uso de lombricompost de estiércol vacuno se obtuvieron los mejores resultados, particularmente cuando se aplicó a $25 \%$ en el sustrato; en contraste, el desarrollo fue pobre cuando se utilizó compost vacuno y abono comercial en su proporción más alta. Además de estar acorde con las recomendaciones en términos de las propiedades fisicoquímicas y biológicas, los resultados obtenidos al aplicar vermicompost pueden deberse a que este material posee una gran riqueza de microorganismos, reducidas cantidades de sales solubles, además de macro y micronutrientes en diferentes proporciones; incluso tiene la capacidad de aumentar poblaciones bacterianas cuando se mezcla con el suelo (Durán y Henríquez 2007, Fraile y Obando 1994, Moreno et al. 2005, Tognetti et al. 2013).

Por último, podemos indicar que las características finales de los lombricompost están determinadas por la naturaleza de las fuentes orgánicas utilizadas para su elaboración (Durán y Henríquez 2007) y pueden estar asociadas con las respuestas observadas en cada especie cultivada.

\section{Conclusiones}

Los efectos de los abonos orgánicos aplicados al sustrato de crecimiento, sobre el crecimiento y floración de plantas de geranio y belén, están asociados al tipo de abono, la proporción aplicada, la especie y las características de los abonos usados. El lombricompost elaborado con estiércol vacuno en proporciones de $25 \%$ en el sustrato resultó con efectos similares a los de la tierra de hoja, por los efectos benéficos que genera en crecimiento, número de hojas y longitud de raíz en geranio, así como en crecimiento y floración en geranio y belén, por lo que este tipo de abono se recomienda en el sustrato de crecimiento, como alternativa en sustitución de la tierra de hoja comúnmente usada en viveros y traspatios comunitarios.

El abono comercial y el compost de estiércol vacuno a proporciones superiores a $50 \%$ en el sustrato generaron efectos negativos en el crecimiento $y$ floración de geranio y belén, efectos relacionados con la proporción aplicada, las características de los abonos usados y la especie ornamental. 


\section{LITERATURA CITADA}

Ansorena-Miner [internet]. 2010. La red de compostaje doméstico en Gipuzkoa. [cited 2014 Mar 14]. Disponible en: http://www.compostaenred.org/proyectos/JonadasNavarra2010/Ponencias/J\%20ANSORENA.pdf

Asciutto K, Rivera MC, Wright ER, Morisigue D, López MV. 2006. Effect of vermicompost on the growth and health of Impatiens wallerana. Phyton. Revista Internacional de Botánica Experimental 75: 115-123.

Atiyeh RM, Edwards CA, Subler S, Metzger JD. 2001. Pig manure vermicompost as a component of a horticultural bedding plant medium: effects on physicochemical properties and plant growth. Bioresource Technology 78: 11-20. https://doi.org/10.1016/S0960-8524(00)00172-3

Borji S, Khodadadi M, Mobasser HR. 2014. Effect of different levels of vermicompost on growth characteristics and flowering geranium. Journal of Novel Applied Sciences 3(3): 307-309.

Chamani E, Joyce DC, Reihanytanbar A. 2008. Vermicompost effects on the of growth and flowering of Petunia hybrida 'Dream Neon Rose. American-Eurasian Journal of Agriculture and Environmental Sciences 3(3): 506512.

Durán L, Henríquez C. 2007. Caracterización química, física y microbiológica de vermicompostes producidos a partir de cinco sustratos orgánicos. Agronomía Costarricense 31(1): 41-51.

[FAO] Food and Agriculture Organization of the United Nations. 2006. Livestock's long shadow environmental issues and options. Rome, Italia. 390 pp.

Fraile J, Obando R. 1994. Lombricultura: alternativa para el manejo racional de los desechos del banano. Aqua 3(4): 17-22.

García-Albarado JC, Trejo-Téllez LI, Velásquez-Hernández MA, Ruiz-Bello A, Gómez-Merino FC. 2010. Crecimiento de petunia en respuesta a diferentes proporciones de composta en sustrato. Revista Chapingo Serie Horticultura 16: 107-113.

García-Gomez A, Bernal MP, Roig A. 2002. Growth of ornamental plants in two composts prepared from agroindustrial wastes. Bioresource Technology 83(2): 81-87. https://doi.org/10.1016/S0960-8524(01)00211-5

Grigatti M, Giorgioni ME, Ciavatta C. 2007. Compost-based growing media: Influence on growth and nutrient use of bedding plants. Bioresource Technology 98(18): 3526-3534. https://doi.org/10.1016/j.biortech.2006.11.016

Guarneros-Zarandona N, Morales-Jiménez J, Cruz-Hernández J, Huerta-Peña A, Ávalos-Cruz DA. 2014. Economía familiar e índice de biodiversidad de especies en los traspatios comunitario de Santa María Nepopualco, Puebla. Revista Mexicana de Ciencias Agrícolas 9: 17011712.

Íñiguez G, Crohn DM. 2010. Fertilization of potted geranium with a slaughterhouse. In: Íñiguez CG, editor. Experiencias en el compostaje de residuos industriales y pecuarios. Guadalajara, Universidad de Guadalajara-CUCEI. p: 91-101.
Joshi R, Singh J, Vig AP. 2015. Vermicompost as an effective organic fertilizer and biocontrol agent: effect on growth, yield and quality of plants. Reviews in Environmental Science and Bio/Technology 14(1): 137-159. https:// doi.org/10.1007/s11157-014-9347-1

Lazcano C, Domínguez J. 2010. Effects of vermicompost as a potting amendment of two commercially-grown ornamental plant species. Spanish Journal of Agricultural Research 8(4): 1260-1270. https://doi.org/10.5424/ sjar/2010084-1412

Moreno A, Valdés MT, Zarate T. 2005. Desarrollo de tomate en sustratos de vermicompost/arena bajo condiciones de invernadero. Agricultura Técnica 65(1): 26-34. https:// doi.org/10.4067/S0365-28072005000100003

Pinos-Rodríguez JM, García-López JC, Peña-Avelino LY, Rendón-Huerta JA, González-González C, Tristán-Patiño F. 2012. Impactos y regulaciones ambientales del estiércol generado por los sistemas ganaderos de algunos países de América. Agrociencia 46(4): 359-370.

Preston TR. 2005. Ventajas de los animales pequeños en los sistemas agropecuarios. LEISA 21(3): 5-7.

Montgomery DC. 2011. Diseño y análisis de experimentos. México, DF.

Ramos AD, Terry AE. 2014. Generalidades de los abonos orgánicos: importancia del bocashi como alternativa nutricional para suelos y plantas. Cultivos Tropicales 35(4): 52-59.

Santamaría-Romero S, Ferrera-Cerrato R, Almaraz-Suárez JJ, Galvis-Spinola A, Barois-Boullard I. 2001. Dinámica y relaciones de microorganismos, C-orgánico y N-total durante el composteo y vermicomposteo. Agrociencia 35: 377-384.

Tognetti C, Laos L, Mazzarino MJ, Hernández MT. 2013. Composting vs. vermicomposting: A comparison of end product quality. Compost Science and Utilization 13(1): 6-13. https://doi.org/10.1080/1065657X.2005.10702212

Velásquez JC, León J. 2006. CEDICAM: Una organización de campesinos para campesinos en México. LEISA Revista de Agroecología 22(2): 24-26.

Vieyra J, Castillo A, Losada H, Cortés J, Alonso G, Ruiz T, Hernández P, Zamudio A, Acevedo A. 2004. La participación de la mujer en la producción de traspatio y sus beneficios tangibles e intangibles. Cuadernos de Desarrollo Rural 53: 9-23. 